\title{
INFORMAÇÕES ESPECTROELETROMAGNÉTICAS E TOPOGRÁFICAS NA DETERMINAÇÃO DE TEORES DE COMPONENTES DO SOLO ${ }^{\left({ }^{1}\right)}$
}

\author{
ALINE MARQUES GENÚ $\left({ }^{*}\right)$; JOSÉ ALEXANDRE M. DEMATTÊ $\left({ }^{3}\right)$
}

\begin{abstract}
RESUMO
A obtenção de informações quantitativas do solo por técnicas não destrutivas, como o sensoriamento remoto e os modelos digitais do terreno (MDT), abre a possibilidade de avaliações mais rápidas e menos onerosas no estudo de solos. O objetivo deste trabalho foi verificar a possibilidade de quantificar atributos do solo utilizando informações extraídas de sensor orbital ASTER conjugados com parâmetros topográficos. A área de estudo com 184 ha, localizada em Rafard (SP), foi amostrada em forma de grid sendo coletada uma amostra por hectare. A reflectância foi obtida da imagem ASTER e os dados topográficos a partir do MDT. Em seguida, utilizando os dados orbitais e de relevo foram gerados os modelos de regressão para os atributos do solo. As variáveis dependentes foram selecionadas a partir do método stepwise. Posteriormente, os modelos com $\mathrm{R}^{2}>0,50$ foram testados em amostras desconhecidas de terra e os valores estimados pelas equações (VE) e os valores determinados em laboratório (VD) foram avaliados através de gráficos de dispersão. Os componentes $\mathrm{SiO}_{2}, \mathrm{Fe}_{2} \mathrm{O}_{3}, \mathrm{TiO}_{2}$ e argila foram os atributos testados, e $\mathrm{Fe}_{2} \mathrm{O}_{3}$ e argila obtiveram os melhores coeficientes de determinação. Conclui-se que os modelos gerados pelos dados espectrais e de relevo permitem quantificar os referidos componentes do solo.
\end{abstract}

Palavras-chave: modelo digital do terreno, imagem ASTER, atributos do solo.

\section{ABSTRACT \\ SPECTRALELETROMAGNETIC AND TOPOGRAPHIC INFORMATIONS ON THE DETERMINATION OF SOIL COMPOUNDS}

The obtention of soil quantitative information by non destructive techniques, like remote sensing and digital elevation model (DEM) open a possibility of faster and cheaper evaluation on soil studies. This study aims at verifying the possibility of quantifying soil atributtes through ASTER orbital sensor data and topographic features. The study area, with 184 ha, located in Rafard County, São Paulo State, Brazil, was sampled in a grid way collecting 1 sample/ha. Reflectance data was obtained from ASTER image and topographic features from DEM. Then, using orbital an topographic data were generated a multiple regression equation for soil attributes. The dependent variables were selected by a forward stepwise method. After that, the models with $\mathrm{R}^{2}>0.50$ were tested with unknown soil samples and the estimated values (VE) and the determined values (VD) were evaluated through scatterplots. $\mathrm{SiO}_{2}, \mathrm{Fe}_{2} \mathrm{O}_{3}$, $\mathrm{TiO}_{2}$ and clay attributes were tested and, $\mathrm{Fe}_{2} \mathrm{O}_{3}$ and clay obtained higher values of determination coefficient. It is conclude that the models generated with spectral data and topographic information allowed the quantification of the above cited soil components.

Key words: digital elevation model (DEM), ASTER image, soil attributes.

$\left({ }^{1}\right)$ Recebido para publicação em 13 de novembro de 2008 e aceito em $1 .^{\circ}$ de setembro de 2009.

${ }^{(2)}$ Departamento de Agronomia, UNICENTRO. Rua Simeão Camargo Varela de Sá, 03, 85040-080 Guarapuava (PR). E-mail: agenu@unicentro.br. $\left(^{*}\right)$ Autora correspondente.

$\left(^{3}\right)$ Departamento de Ciência do solo, ESALQ/USP, Caixa Postal 09, 13418-900 Piracicaba (SP). E-mail: jamdemat@esalq.usp.br 


\section{INTRODUÇÃO}

Existe uma demanda cada vez maior por informações quantitativas para fins de levantamento e mapeamento, monitoramento ambiental, estratégias de manejo entre outros (SCULL et al., 2003; MCBRATNEY et al., 2006). Especificamente em relação a solos, o principal método para a geração destas informações é a análise de solo convencional realizada em laboratório. Entretanto, este método é caro e demorado para as necessidades atuais. Assim, novas técnicas que tornem o processo de avaliação do solo mais rápido, menos oneroso e com menor risco ambiental vêm sendo buscadas (UDELHOVEN et al., 2003).

A espectrometria de reflectância em laboratório tem sido aceita como um método rápido, não destrutivo e que permite a partir de um único espectro, a caracterização de vários constituintes do solo (VISCARRa Rossel et al., 2006). A reflectância do solo, em todas as faixas do espectro eletromagnético, é uma propriedade cumulativa derivada do comportamento espectral combinado dos minerais, água e matéria orgânica (UDELHOVEN et al., 2003).

A análise espectral quantitativa dos solos requer técnicas estatísticas para discriminar a resposta dos atributos do solo das características espectrais (VISCARRa Rossel et al., 2006). Vários métodos têm sido utilizados para relacionar o espectro do solo com seus atributos como, por exemplo, regressão múltipla (DEMATTÊ E NANNI, 2003), componentes principais (CHANG et al., 2001) e redes neurais (DANIEL et al., 2003).

Existe na literatura grande número de trabalhos sobre a utilização de dados espectrais de laboratório e análises estatísticas para a quantificação de atributos do solo, conforme pode ser observado no trabalho de Viscarra Rossel et al. (2006), o qual fornece um levantamento sobre o tema. Entretanto, segundo Scull et al. (2003) e Huete (2004) poucos trabalhos tratam da utilização de sensores orbitais e aerotransportados.

Outra forma de estudo do solo é a utilização dos Modelos Digitais do Terreno (MDT). Segundo Moore et al. (1993), a análise do terreno possibilita quantificar os componentes do relevo que afetam o desenvolvimento do solo e, consequentemente, a distribuição espacial dos seus atributos. Por isso, vários pesquisadores (MOORE et al., 1993; Dobos et al., 2000; Lagacherie e Voltz, 2000) vêm utilizando informações de elevação, declividade, orientação de vertentes e forma (concavidade e convexidade) como variáveis auxiliares na caracterização, mapeamento e predição de propriedades do solo.
Como se observa, existem duas formas de determinar quantitativamente componentes do solo: pelo sensoriamento espectral e por dados topográficos. Não se verifica, entretanto, pesquisas que unem essas duas formas de análise quantitativa, verificando sua potencialidade no estudo do solo.

Devido à correlação existente entre a radiação eletromagnética e os atributos do solo e do modelo digital do terreno (MDT) com a distribuição espacial dos solos e suas propriedades, espera-se que a informações espectrais conjugadas a dados topográficos permitam quantificar componentes físicos do solo tais como argila e ferro. Desta forma, a finalidade desta pesquisa foi verificar a utilização destas duas informações combinadas na quantificação das bases trocáveis (Ca e Mg), soma de bases (S), acidez potencial $(\mathrm{H}+\mathrm{Al})$, matéria orgânica $(\mathrm{MO})$, capacidade de troca catiônica (CTC), argila, areia, silício total $\left(\mathrm{SiO}_{2}\right)$, ferro total $\left(\mathrm{Fe}_{2} \mathrm{O}_{3}\right)$ e titânio total $\left(\mathrm{TiO}_{2}\right)$.

\section{MATERIAL E MÉTODOS}

A área de estudo localiza-se no município de Rafard, sudoeste do Estado de São Paulo. É delimitada pelas coordenadas geográficas $23^{\circ} 0^{\prime} 31,37^{\prime \prime}$

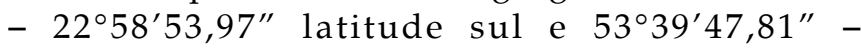
$53^{\circ} 37^{\prime} 25,65^{\prime \prime}$ longitude oeste. O clima da região é classificado como mesotérmico de inverno seco em que a temperatura média do mês mais frio é inferior a 18 ${ }^{\circ} \mathrm{C}$ e a do mês mais quente ultrapassa $22{ }^{\circ} \mathrm{C}$ (Centro Nacional de Ensino e Pesquisa Agronômica, 1960).

A geologia da região pertence à formação Itararé, Grupo Tubarão (IPT, 1981) contituindo-se de arenitos de granulação heterogênea, argilitos e folhelhos de colorações variadas desde o cinza-claro ao escuro. A área possui ainda basaltos da formação Serra Geral, Grupo São Bento e, próximos ao rio, seixos. O relevo em sua maior parte é caracterizado como ondulado e suave ondulado, sendo o primeiro de maior área, com altitude variando entre 475 e 565 metros sobre o nível do mar.

Foram utilizados dados de 184 pontos de tradagem georreferenciados e a coleta de amostras na profundidade de $0-20 \mathrm{~cm}$. Depois de coletadas, as amostras foram peneiradas ( $2 \mathrm{~mm}$ ) e em seguida secas em estufa a $50{ }^{\circ} \mathrm{C}$ por 48 horas. Posteriormente, na fração menor que $2 \mathrm{~mm}$ foram efetuadas as análises físicas e químicas.

Para a análise física, foram determinados os teores de areia, silte e argila a partir do método do densímetro (CAMARGo et al., 1986). Para as análises químicas, determinaram-se $\mathrm{pH}$ (água e $\mathrm{KCl}$ ), $\mathrm{Ca}^{2+}$, 
$\mathrm{Mg}^{2+}, \mathrm{Al}^{3+}, \mathrm{H}+\mathrm{Al}$ e matéria orgânica (MO), de acordo com Raij e Quaggio (1989). A partir desses dados, obteve-se o valor da soma de bases (S), capacidade de troca catiônica (CTC), saturação por bases $(\mathrm{V} \%)$ e saturação por alumínio $(\mathrm{m} \%)$. O ferro total $\left(\mathrm{Fe}_{2} \mathrm{O}_{3}\right)$, a sílica $\left(\mathrm{SiO}_{2}\right)$ e o titânio $\left(\mathrm{TiO}_{2}\right)$ foram determinados pelo ataque sulfúrico, através de metodologia descrita por CAMARgo et al. (1986).

Os dados espectrais foram extraídos de oito bandas do sensor ASTER assim designadas: B1 (verde, 520-600 nm), B2 (vermelho, 630-690 nm), B3 (infravermelho próximo, $760-860 \mathrm{~nm}$ ) e cinco bandas do infravermelho médio B4 (infravermelho médio, 1600-1700 nm), B5 (infravermelho médio, 2145-2185 $\mathrm{nm}$ ), B6 (infravermelho médio, 2185-2225 nm), B7 (infravermelho médio, 2235-2285 nm), B8 (infravermelho médio, 2295-2365 nm). A data da imagem é de 6 de agosto de 2004, a resolução espacial de 30 metros e as coordenadas do centro da cena são $22^{\circ} 42^{\prime} 33^{\prime \prime}$ latitude sul e $48^{\circ} 00^{\prime} 45^{\prime \prime}$ longitude oeste.

Para a conversão dos níveis de cinza da imagem para reflectânica e para eliminar o efeito da atmosfera na imagem de satélite utilizou-se o programa ACORN (Atmospheric Correction Now) (AIG, 2001).

Para a coleta dos dados espectrais orbitais, a informação da posição dos 184 pontos de amostragem foi sobreposta às composições coloridas 3R4G2B e 4R3G2B da imagem utilizada. Posteriormente, para certificar-se de que os pontos estavam sobre área de solo exposto, gerou-se uma imagem de índice de vegetação NDVI (JACKSON e HUETE, 1991) e utilizou-se o método da linha do solo (BARRET et al., 1993).

O Modelo Digital do Terreno foi gerado no programa ArcGIS 9 (EsRI, 2005) usando como base as curvas de nível da área, espaçadas de 5 em $5 \mathrm{~m}$ obtidas das cartas planialtimétricas de Toledos e Costa Rica, ambas na escala 1:10.000 e o limite da área de trabalho. Posteriormente, a partir do MDT, geraramse os mapas de declividade e de orientação de vertentes. Para declividade utilizaram-se as classes de relevo existentes em EMBRAPA (2006): plano (0 a 3\%), suave ondulado $(3 \%$ a $8 \%)$, ondulado $(8 \%$ a $20 \%)$, forte ondulado (20\% a $45 \%)$, montanhoso $(45 \%$ a $75 \%$ ) e escarpado (acima de $75 \%$ ). Para orientação de vertentes, utilizaram-se as classes (ALVES, 2005): norte $\left(0\right.$ a $\left.45^{\circ}\right)$, nordeste $\left(45\right.$ a $\left.90^{\circ}\right)$, leste $\left(90\right.$ a $\left.135^{\circ}\right)$, sudeste $\left(135\right.$ a $\left.180^{\circ}\right)$, sul $\left(180\right.$ a $\left.225^{\circ}\right)$, sudoeste $\left(225\right.$ a $\left.270^{\circ}\right)$, oeste $\left(270\right.$ a $\left.315^{\circ}\right)$ e noroeste $\left(315\right.$ a $\left.360^{\circ}\right)$. Desta forma, após a geração do MDT, do mapa de declividade e do mapa de orientação de vertentes, o arquivo dos pontos de tradagem foi sobreposto às imagens, obtendo - se assim as informações de relevo para cada amostra de solo.
Para geração dos modelos de estimativa de atributos, utilizaram-se às informações das bandas do sensor ASTER, elevação, declividade e orientação de vertentes de 75 amostras de solos como variáveis dependentes e, as 109 amostras restantes, foram utilizadas na validação das equações.

Todas as informações sobre os atributos químicos e físicos, valores de reflectância nas oito bandas do sensor ASTER e os dados de elevação, declividade e orientação de vertentes dos pontos amostrais selecionados para a construção dos modelos estatísticos foram inseridos no programa Statistical Analysis System (SAS, 1999). Utilizou-se o procedimento proc reg para a geração do modelo de regressão múltipla, juntamente com a função stepwise para a seleção das variáveis independentes que fariam parte do modelo para cada atributo. Inicialmente, obtevese o modelo apenas para os dados orbitais e depois com as informações orbitais e topográficas juntas.

Posteriormente, os atributos cujos modelos de regressão múltipla obtiveram $R^{2}$ maior que 0,5 , foram validados utilizando-se as 109 amostras não utilizadas na geração do modelo. Os valores obtidos na validação das equações de regressão múltipla foram denominados teores estimados (VE). Em seguida, para verificar se os dados estimados tinham relação com os dados determinados em laboratório (VD), gerou-se um gráfico de dispersão entre eles.

\section{RESULTADOS E DISCUSSÃO}

\section{Quantificação de teores de atributos do solo por satélite}

Os elementos para os quais foram obtidas equações de regressão múltipla estão relacionados na tabela 1. Para os demais atributos determinados em laboratório: silte, $\mathrm{pH}$ (água, $\mathrm{KCl}$ ), $\mathrm{Al}^{+3}, \mathrm{~V} \%$ e m\%, não foi possível obter um modelo, pois nenhuma variável independente foi estatísticamente significativa a 5\%.

Nas equações relativas aos elementos obtidos através do ataque sulfúrico, ou seja, $\mathrm{SiO}_{2}, \mathrm{Fe}_{2} \mathrm{O}_{3}$ e $\mathrm{TiO}_{2}$, o coeficiente de determinação foi de 0,48, 0,65 e 0,66 para cada atributo respectivamente, ressaltando que o valor para titânio foi o maior obtido dentre os atributos estudados (Tabela 1). Fiorio (2002), trabalhando com solos da região de Barra Bonita e imagem do satélite TM-Landsat 5 obteve coeficientes de determinação de $0,6531,0,6724$ e 0,7210 para os mesmos atributos. Já Nanni e Demattê (2006), utilizando imagem TM obtiveram $\mathrm{R}^{2}$ de $0,598,0,725$ e 0,727 e para sensor em laboratório obtiveram coeficientes ainda mais altos, em torno de 0,9 para estes elementos. 
Tabela 1. Equações de regressão múltipla para estimativa dos atributos do solo usando dados espectrais e topográficos

\begin{tabular}{|c|c|c|}
\hline Atributo & Modelo $\left({ }^{1}\right)$ & $R^{2}\left({ }^{2}\right)$ \\
\hline & Aster & \\
\hline $\mathrm{Ca}\left({ }^{3}\right)$ & $\sqrt{\mathrm{Ca}}=24,85+(125,2 * \mathrm{~B} 1)+(32 * \mathrm{~B} 4)+(-176,6 * \mathrm{~B} 6)+\left(-81,22^{*} \mathrm{~B} 8\right)$ & 0,47 \\
\hline $\operatorname{Mg}\left({ }^{4}\right)$ & $\mathrm{Mg}=15,58+\left(230,03^{*} \mathrm{~B} 4\right)+\left(251,81^{*} \mathrm{~B} 6\right)+\left(-554,51^{*} \mathrm{~B} 8\right)$ & 0,34 \\
\hline $\mathrm{H}+\mathrm{Al}\left(\left(^{5}\right)\right.$ & $\mathrm{HAl}=46,30+\left(-206,7^{*} \mathrm{~B} 2\right)+(850,9 * \mathrm{~B} 3)+(-557,3 * \mathrm{~B} 4)$ & 0,23 \\
\hline $\mathrm{MO}\left({ }^{6}\right)$ & $\mathrm{MO}=36,10+(-110,79 * \mathrm{~B} 5)$ & 0,20 \\
\hline$S\left({ }^{7}\right)$ & $\mathrm{S}=40,59+\left(-584,73^{*} \mathrm{~B} 1\right)+\left(1089,18^{*} \mathrm{~B} 4\right)+(945,52 * \mathrm{~B} 6)+\left(-2001,93^{*} \mathrm{~B} 8\right)$ & 0,43 \\
\hline $\mathrm{CTC}\left({ }^{8}\right)$ & $\mathrm{CTC}=154,65+(991,74 * \mathrm{~B} 2)+(-956,42 * \mathrm{~B} 3)+(973,80 * \mathrm{~B} 4)+(1031,94 * \mathrm{~B} 6)+(-2267,81 * \mathrm{~B} 8)$ & 0,51 \\
\hline $\mathrm{SiO}_{2}\left({ }^{9}\right)$ & $\mathrm{LogSiO}_{2}=2,43+\left(-9,97^{*} \mathrm{~B} 1\right)+(9,32 * \mathrm{~B} 2)+\left(3,36^{*} \mathrm{~B} 4\right)+(-8,39 * \mathrm{~B} 5)$ & 0,48 \\
\hline $\mathrm{Fe}_{2} \mathrm{O}_{3}\left({ }^{10}\right)$ & $\operatorname{LogFe}_{2} \mathrm{O}_{3}=2,96+\left(-20,03^{*} \mathrm{~B} 1\right)+\left(10,16^{*} \mathrm{~B} 2\right)+\left(-2,59^{*} \mathrm{~B} 8\right)$ & 0,65 \\
\hline $\mathrm{TiO}_{2}\left({ }^{11}\right)$ & $\mathrm{LogTiO}_{2}=2,11+\left(-20,73^{*} \mathrm{~B} 1\right)+\left(10,23^{*} \mathrm{~B} 2\right)+\left(-1,49^{*} \mathrm{~B} 8\right)$ & 0,66 \\
\hline Argila & Argila $=687,46+(-2078,20 * B 8)$ & 0,36 \\
\hline \multirow[t]{2}{*}{ Areia } & Areia $=-78,08+\left(-3012,38^{*} \mathrm{~B} 2\right)+\left(4800,88^{*} \mathrm{~B} 8\right)$ & 0,36 \\
\hline & Aster + Topografia & \\
\hline $\mathrm{Ca}$ & $-\left({ }^{12}\right)$ & - \\
\hline $\mathrm{Mg}$ & - & - \\
\hline $\mathrm{H}+\mathrm{Al}$ & - & - \\
\hline $\mathrm{MO}$ & $\mathrm{MO}=68,58+\left(-102,85^{*} \mathrm{~B} 5\right)+\left(-0,07^{*} \mathrm{EL}\right)$ & 0,25 \\
\hline S & - & - \\
\hline CTC & - & - \\
\hline $\mathrm{SiO}_{2}$ & $\begin{array}{l}\mathrm{SiO}_{2}=24,91+\left(-1301,66^{*} \mathrm{~B} 1\right)+\left(1890,66^{*} \mathrm{~B} 2\right)+\left(-891,89^{*} \mathrm{~B} 3\right)+\left(1222,74^{*} \mathrm{~B} 4\right)+\left(-1124,56^{*} \mathrm{~B} 5\right) \\
+\left(735,81^{*} \mathrm{~B} 6\right)+\left(-1243,10^{*} \mathrm{~B} 8\right)+\left(0,28^{*} \mathrm{EL}\right)+\left(-0,04^{*} \mathrm{AS}\right)\end{array}$ & 0,58 \\
\hline $\mathrm{Fe}_{2} \mathrm{O}_{3}$ & $\operatorname{LogFe}_{2} \mathrm{O}_{3}=2,12+\left(-21,45^{*} \mathrm{~B} 1\right)+\left(10,54^{*} \mathrm{~B} 2\right)+\left(-2,42^{*} \mathrm{~B} 8\right)+\left(0,0020^{*} \mathrm{EL}\right)+\left(-0,0004^{*} \mathrm{AS}\right)$ & 0,68 \\
\hline $\mathrm{TiO}_{2}$ & $\left(\mathrm{TiO}_{2}\right)^{-0,3}=-0,30+\left(9,14^{*} \mathrm{~B} 1\right)+\left(-4,29^{*} \mathrm{~B} 2\right)+\left(0,0008^{*} \mathrm{EL}\right)+\left(0,0001^{*} \mathrm{AS}\right)$ & 0,66 \\
\hline Argila & Argila $=109,39+\left(-5473,10^{*} \mathrm{~B} 1\right)+\left(5137,16^{*} \mathrm{~B} 2\right)+\left(-2353,59^{*} \mathrm{~B} 8\right)+\left(1,18^{*} \mathrm{EL}\right)+\left(-0,14^{*} \mathrm{AS}\right)$ & 0,52 \\
\hline Areia & Areia $=-133,03+\left(-2988,14^{*} \mathrm{~B} 2\right)+(4871,70 * \mathrm{~B} 8)+(0,22 * \mathrm{AS})$ & 0,38 \\
\hline
\end{tabular}

$\left({ }^{1}\right)$ B1...B8: Bandas ASTER; EL: elevação; AS: orientação da vertente. $\left({ }^{2}\right)$ Coeficiente de determinação. $\left({ }^{3}\right)$ Cálcio. $\left({ }^{4}\right)$ Magnésio. $\left({ }^{5}\right)$ Acidez potencial. $\left({ }^{6}\right)$ Matéria Orgânica. $\left({ }^{7}\right)$ Soma de Bases. $\left({ }^{8}\right)$ Capacidade de troca catiônica. $\left({ }^{9}\right)$ Silício Total. $\left({ }^{10}\right)$ Ferro Total. $\left({ }^{11}\right)$ Titânio Total. $\left({ }^{12}\right)$ Não foram gerados modelos significativos com dados orbitais e topográficos.

Nos modelos gerados para ferro e titânio total, as bandas 1,2 e 8 foram selecionadas em ambos os casos (Tabela 1). As bandas 1 e 2 referem-se à faixa do visível, verde (520-600 nm) e vermelho (630-690 $\mathrm{nm}$ ), nestes comprimentos de onda ocorrem algumas das bandas de absorção do ferro como em $550 \mathrm{~nm}$ pelo $\mathrm{Fe}^{2+}$ ou estão muito próximas a elas como do $\mathrm{Fe}^{2+} \mathrm{em}$ $510 \mathrm{~nm}$ e do $\mathrm{Fe}^{3+}$ em 700 nm. O titânio, por sua vez, pode estar associado ao ferro na ilmenita $\left(\mathrm{FeTiO}_{3}\right)$ ou na forma dos minerais anatásio e rutilo que, também, podem conter ferro na sua estrutura como impureza; assim, a feição de absorção do titânio no visível acaba tendo uma aparência muito próxima à observada para o ferro (Meneses e Ferreira Junior, 2001).

A banda 8, na faixa do infravermelho médio, também corresponde às bandas de absorção em torno de 2000 a 2500 nm, devido à ligação metal-OH, no qual o metal pode ser Fe e Ti, entre outros (MENESES e FERREIRA JUNIOR, 2001).
Com relação ao silício, a grande maioria dos minerais existentes no solo possui este elemento em sua estrutura. Além disso, sabe-se que o quartzo, composto basicamente de silício, apesar de não ter feições de absorção do visível ao infravermelho, possui alta intensidade de reflectância nesta faixa (BEN-Dor et al., 1999). Diante disso, esperava-se que todas as bandas ASTER fossem inseridas no modelo e não apenas quatro, as duas do visível (1 e 2) e duas do infravermelho médio (4 e 5).

Verifica-se, entretanto, que os elementos do ataque sulfúrico apresentam resultados consistentes, com coeficientes em geral acima de 0,5 , demonstrando que é possível estimar estes atributos através de sensores orbitais e terrestre. Isso se deve ao fato de estes elementos fazerem parte da mineralogia do solo, o que tem grande efeito na resposta espectral dos solos, principalmente na faixa do visível e infravermelho. Vale ressaltar que, dentre os três, a 
sílica foi o que obteve menores coeficientes em todos os trabalhos.

Para os atributos granulométricos, argila e areia, o coeficiente foi de $0,36 \mathrm{em}$ ambos os casos (Tabela 1). Estes valores foram considerados baixos e discordam dos obtidos por SousA Junior (2005) que trabalhando com imagem ASTER na região de Ibaté e São Carlos, obteve coeficientes de determinação de 0,56 para argila e 0,52 para areia. FIORIO (2002), utilizando imagem Landsat 5, obteve um $\mathrm{R}^{2}$ de 0,6140 para argila e 0,6356 para areia, enquanto SulLivan et al. (2005), utilizando um sensor aerotransportado obtiveram 0,42 para areia e 0,59 para argila.

Poucas bandas foram selecionadas nos modelos de areia e argila, estando a banda 8 presente em ambos os casos, pois se relaciona com a mineralogia do solo, especialmente aos minerais de argila (Meneses e FerReIRA Junior, 2001). Entretanto, esperava-se que a banda 7 também estivesse presente, pois é a que mais se relaciona com a faixa conhecida para as absorções da caulinita e da gibsita.

A matéria orgânica possui baixo valor de $\mathrm{R}^{2}$ com 0,20 , semelhante ao obtido por SousA JUNIOR (2005) de 0,32 , utilizando imagem ASTER e ao de COLEMAN et al. (1993) com 0,108 trabalhando com imagem TM. Nanni e Demattê (2006), utilizando sensor em laboratório obtiveram $\mathrm{R}^{2}$ de 0,797 , enquanto SousA JUNIOR (2005), também com sensor em laboratório, obteve 0,38 .

A matéria orgânica tem grande influência na curva espectral do solo, por isso, espera-se que os modelos estatísticos sejam capazes de estimar este atributo de forma eficiente. No caso deste trabalho, apenas a banda 5 foi selecionada, que corresponde à faixa do infravermelho médio que vai de 2145 a 2185 nm; como a matéria orgânica possui vários grupos funcionais, relacionados a diversas feições de absorção (BEN-Dor et al., 1999), talvez uma destas feições esteja presente na faixa recoberta pela banda 5 .

Por outro lado, Henderson et al. (1992) verificaram que as diferentes fases de decomposição da matéria orgânica têm respostas espectrais distintas em todo espectro eletromagnético, fato que pode ser a causa da variabilidade dos coeficientes de determinação obtidos pelos diferentes sensores.

Com relação aos atributos químicos, cálcio e soma de bases proporcionaram $\mathrm{R}^{2}$ de 0,47 e 0,43 , respectivamente e a CTC de 0,51 (Tabela 1 ). Já o magnésio e a acidez obtiveram coeficientes menores $(0,34$ e 0,23 respectivamente). SousA JUNIOR (2005) obteve 0,47 para cálcio e 0,52 para CTC e soma de bases, utilizando imagem ASTER. Coeficientes similares foram verificados por NANNI e Demattê (2006) e Fiorio (2002) com imagens TMLandsat. No caso dos sensores terrestres CHANG et al. (2001), Shepherd e Walsh (2002) e NANni e Demattê (2006) obtiveram valores de $\mathrm{R}^{2}$ acima de 0,7 para cálcio e CTC.

Nota-se, nos modelos gerados para os atributos químicos que, praticamente, todos possuem as bandas 4,6 e 8, na faixa do infravermelho médio, na qual ocorre maior influência da mineralogia e da matéria orgânica do solo, que são as principais fontes das cargas do solo e, desta forma, atuam sobre suas propriedades químicas avaliadas neste trabalho. A CTC representa a quantidade de íons positivos que podem ser retidos no solo por atração eletrostática, ou seja, por atração de cargas elétricas opostas e, os principais cátions trocáveis que podem ser atraídos são cálcio, magnésio, potássio, sódio, alumínio e hidrogênio (RAIJ et al., 2001).

\section{Quantificação de teores de atributos do solo por} dados de satélite e topográficos

Com o intuito de verificar a utilização de informações de relevo na quantificação de atributos do solo, foram inseridas nos modelos espectrais, como variáveis independentes, a elevação, a declividade e a orientação de vertentes.

Para os atributos químicos magnésio, cálcio, acidez potencial $(\mathrm{H}+\mathrm{Al})$, soma de bases (S) e CTC, nenhum atributo de relevo foi considerado significativo para entrar no modelo (Tabela 1). Esse fato pode ser efeito das modificações ocorridas devido ao manejo químico e aos processos erosivos ocorrentes na área, visto que se utilizaram amostras da camada superficial para a geração dos modelos.

Os atributos físicos argila e areia, com a inclusão dos dados de relevo, obtiveram modelos com maior coeficiente de determinação. Para argila, elevação e orientação de vertentes elevaram o $R^{2}$ de 0,36 para 0,52 . Já para areia, a orientação de vertentes foi o atributo selecionado e o $\mathrm{R}^{2}$ passou de 0,36 para 0,38 (Tabela 1). Moore et al. (1993), utilizando apenas dados de topografia obtiveram para areia, coeficiente de $0,52 \mathrm{com}$ a declividade fazendo parte do modelo estatístico.

Para estimar matéria orgânica, foi adicionada ao modelo a variável elevação e o coeficiente aumentou de 0,20 para 0,25 (Tabela 1). Moore et al. (1993), utilizando apenas parâmetros de topografia para predizer atributos do solo obtiveram, para matéria orgânica, um $\mathrm{R}^{2}$ de 0,48 incluindo no modelo o fator orientação de vertentes. 
Para os elementos do ataque sulfúrico $\mathrm{SiO}_{2}$, $\mathrm{Fe}_{2} \mathrm{O}_{3}$ e $\mathrm{TiO}_{2}$, os atributos elevação e orientação de vertentes foram inseridos nos modelos e, para o caso do $\mathrm{SiO}_{2}$, isto resultou em aumento do coeficiente de 0,48 para 0,58 , enquanto para o ferro o aumento foi de 0,65 para 0,68 e para $\mathrm{TiO}_{2}$ se manteve o mesmo (Tabela 1).

As propriedades do solo variam lateralmente com a topografia. Uma razão para isso é que a orientação da vertente na qual o solo se forma, afeta o microclima, ou seja, a radiação recebida, temperatura do solo e exposição ao vento (PORTA CASANELLAs et al., 1999) com consequente diferenciação no solo.

Nas vertentes mais sombreadas (face sudeste no hemisfério sul), as temperaturas do solo são menores, assim como a evapotranspiração, condicionando ambientes mais úmidos em comparação com as vertentes voltadas para o noroeste, mais ensolaradas à tarde. Resultam destas condições, alterações na intensidade dos processos de hidratação, hidrólise, oxidação e redução, dependendo da direção da face da encosta (Oliveira et al., 1992).

Validação das equações de regressão na quantificação dos atributos dos solos

Para validar as equações de quantificação de atributos, geradas utilizando as informações espectrais e topográficas, foi realizado um gráfico de dispersão entre os valores determinados em laboratório e os valores estimados pelo modelo. Esta análise foi feita para os atributos cujo $\mathrm{R}^{2}=0,5$ a partir de amostras não usadas na geração dos modelos estatísticos.

A argila obteve um coeficiente de determinação de 0,59 (Figura 1a), maior que os constatados por SOUSA JUNIOR (2005), utilizando imagem ASTER de 0,34; FIORIO (2002) com o sensor TM-Landsat 5 que foi de 0,71 e Sullivan et al. (2005), com 0,66. Para os sensores terrestres, BROwn et al. (2006) obtiveram 0,91 e SHEPHERD e WALSH (2002) 0,78.

Para os elementos do ataque sulfúrico $\mathrm{Fe}$, Ti e $\mathrm{Si}$ os coeficientes foram de 0,55, 0,41 e 0,12 respectivamente (Figura 1b,c,d). BROWN et al. (2006), com análise em laboratório, obtiveram para ferro extraído por ditionito $\mathrm{R}^{2}$ de 0,77 e Sullivan et al (2005), usando sensor a bordo de um avião, obtiveram 0,27 também para Ferro ditionito.

Os resultados de validação das equações de quantificação para os atributos argila e ferro total, com $\mathrm{R}^{2}$ maiores que 0,5 , foram considerados satisfatórios utilizando dados ASTER e topográficos, discordando dos verificados por SOUSA JUNIOR (2005) utilizando apenas dados ASTER. Para titânio e silício totais os coeficientes de determinação foram baixos, resultado que pode estar relacionado à grande variabilidade geológica existente na área o que, conseqüentemente, afeta os componentes do solo.

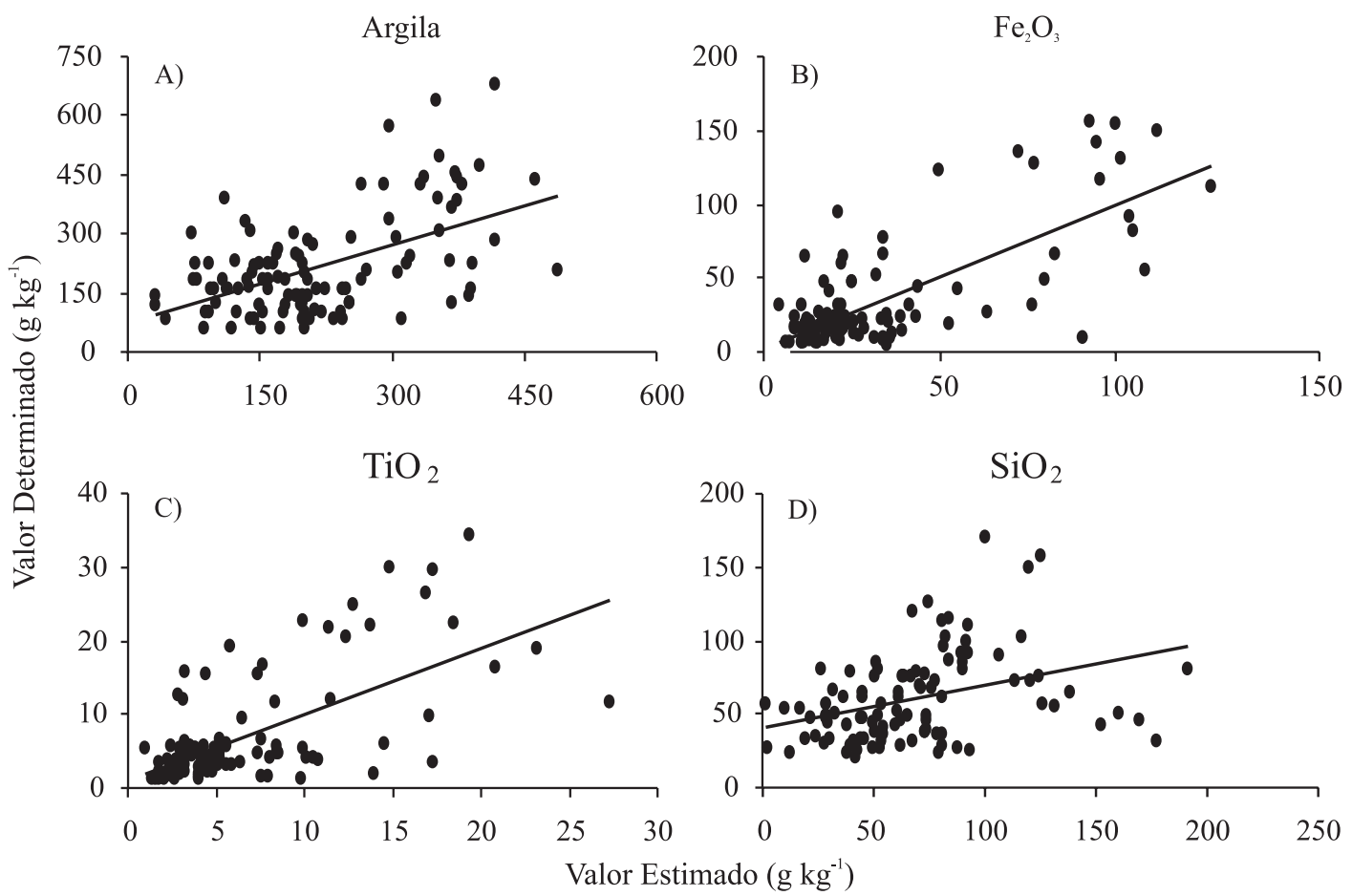

Figura 1. Gráfico de dispersão entre os valores determinados em laboratório e os estimados pelas equações de regressão múltipla para argila (a) y $=0,6696 x+71,372 R^{2}=0,59$; ferro total (b) y $=097744 x+2,6325 R^{2}=0,55$; titânio total (c) y $=0,928 x+1,0824 R^{2}=0,41$; e sílica total $(d) y=0,2707 x+40,953 R^{2}=0,12$. 


\section{CONCLUSÃO}

Os modelos utilizando dados espectrais e topográficos foram melhores que os modelos espectrais para estimar atributos do solo. Os teores de argila e de ferro total, cujas validações obtiveram resultados satisfatórios, podem ser estimados utilizando-se os modelos aqui gerados, entretanto sua aplicabilidade é restrita para a região de realização do trabalho.

\section{REFERÊNCIAS}

ALVES, M.R. Caracterização e uso da bacia hidrográfica do Córrego Zerede, Timóteo-MG. 2005. 95p. Dissertação (Mestrado em Ciência Florestal) - Universidade Federal de Viçosa, Viçosa.

ANALYTICAL IMAGING AND GEOPHYSICS (AIG). Atmospheric correction now (ACORN) 3.12. Boulder, 2001. (1CD-ROM)

BARRET, F.; JACQUEMOUND, S.; HANOCQ, J.F. The soil line concept in remote sensing. Remote Sensing Reviews, v. , n.1, p.1-18, 1993.

BEN-DOR, E.; IRONS, J.R.; EPEMA, G.F. Soil reflectance. In: RENCZ, A.N. (Ed.). Remote sensing for the earth sciences: manual of remote sensing. ${ }^{\text {rd }}$ ed. New York: John Wiley, 1999. p. 111-188.

BROWN, D.J.; SHEPHERD, K.D.; WALSH, M.G.; DEWAYNE MAYS, M.; REINSCH, T.G. Global soil characterization with VNIR diffuse reflectance spectroscopy. Geoderma, v.132, n.3/ 4, p.273-290, 2006.

CAMARGO, A.O.de; MONIZ, A.C.; JORGE, J.A.; VALADARES, J.M. Métodos de análise química, mineralógica e física de solos do IAC. Campinas: IAC, 1986. 94 p. (IAC. Boletim Técnico, 106)

CENTRO NACIONAL DE ENSINO E PESQUISA AGRONÔMICA. Levantamento de reconhecimento de solos do Estado de São Paulo. Rio de Janeiro: Ministério da Agricultura, 1960. 637 p. (SNPA. Boletim 12)

CHANG, C.; LAIRD, D.A.; MAUSBACH, M.J.; HURBURGH JUNIOR, C.R. Near infrared reflectance spectroscopy principal components regression analyses of soil properties. Soil Science Society of America Journal, v.65, p.480-490, 2001.

COLEMAN, T.L.; AGBU, P.A.; MONTGOMERY, O.L. Spectral differenciation of surface soils and soil properties: is it possible from space platforms? Soil Science, v.155, p.283-293, 1993.

DANIEL, K.W.; TRIPATHI, N.K.; HONDA, K. Artificial neural network analysis of laboratory and in situ spectra for the estimation of macronutrients in soils of Lop Buri (Thailand). Australian Journal of Soil Research, v.41, p.47-59, 2003.

DEMATTÊ, J.A.M.; NANNI, M.R. Weathering sequence of soils developed from basalt as evaluated by laboratory (IRIS), airborne (AVIRIS) and orbital (TM) sensors. International Journal of Remote Sensing, v.24, p.4715-4738, 2003.
DOBOS, E.; MICHELI, E.; BAUMGARDNER, M.F.; BIEHL, L.; HELT, T. Use of combined digital elevation model and satellite radiometric data for regional soil mapping. Geoderma, v.97, p.367-391, 2000.

EMBRAPA. Sistema brasileiro de classificação de solos. Brasília: Serviço de Produção de Informação, 2006. 306 p.

ENVIRONMENTAL SYSTEMS RESEARCH INSTITUTE (ESRI). ArcGIS 9.0. Redlands, 2005. (3 CD-ROM)

FIORIO, P.R. Dados radiométricos obtidos nos níveis terrestre e orbital na avaliação de solos. 2002. 198p. Tese (Doutorado em Solos e Nutrição de Plantas) - Escola Superior de Agricultura "Luiz de Queiroz", Universidade de São Paulo, Piracicaba.

HENDERSON, T.L.; BAUMGARDNER, M.F.; FRANZMEIER, D.P.; SCOTT, D.E.; COSTER, D.C. Spectral band selection for classification of soil organic matter content. Soil Science Society of America Journal, v.56, p.865-872, 1992.

HUETE, A. R. Remote sensing of soils and soil processes. In: USTIN, S. (Ed). Remote sensing for natural resources management and environmental monitoring: manual of remote sensing, $3^{\text {rd }}$ ed. Hoboken: John Wiley, 2004. p.3-52.

INSTITUTO DE PESQUISAS TECNOLÓGICAS (IPT). Divisão de Minas e Geologia Aplicada. Mapa geológico de Estado de São Paulo. São Paulo, 1981. Escala 1:1000.000.

JACKSON, R.D.; HUETE, A. R. Interpreting vegetation indices. Preventive Veterinary Medicine, v.11, p.185-200, 1991.

LAGACHERIE, P.; VOLTZ, M. Predicting soil properties over a region using sample information from a mapped reference área and digital elevation data: a conditional probability approach. Geoderma, v.97, p.187-208, 2000.

McBRATNEY, A.C.; MINASNY, B.; VISCARRA ROSSEL, R. Spectral soil analysis and inference systems: a powerful combination for solving the soil data crisis. Geoderma, v.131, p.272-278, 2006.

MENESES, P.R.; FERREIRA JUNIOR, L.G. Comportamento espectral de minerais e rochas. In: MENESES, P.R.; MADEIRA NETTO, J.S. (Org.). Sensoriamento remoto: reflectância de alvo naturais. Brasília: Editora UnB; EMBRAPA Cerrados, 2001. p.63-126.

MOORE, I.D.; GESSLER, P.E.; NIELSEN, G.A.;PETERSON, G.A. Soil attributes prediction using terrain analysis. Soil Science Society of America Journal, v.57, n.6, p.443-452, 1993.

NANNI, M.R.; DEMATTÊ, J.A.M. Spectral methodology in comparison to traditional soil analysis. Soil Science Society of America Journal, v.70, p.393-407, 2006.

OLIVEIRA, J.B.; JACOMINE, P.K.T.; CAMARGO, M.N. Classes gerais de solos do Brasil. Jaboticabal: FUNEP, 1992. 201p.

PORTA CASANELLAS, J.; LOPÉZ-ACEVEDO REGUERÍN, M.; ROQUERO DE LABURU, C. Edafología: para la agricultura y el medio ambiente. Bilbao: Mundi-Prensa, 1999. 849p. 
RAIJ, B. van; QUAGGIO, J.A. Métodos de análise de solo para fins de fertilidade. Campinas: IAC, 1989. 40p. (IAC. Boletim Técnico 8)

RAIJ, B. van; QUAGGIO, J.A.; CANTARELLA, H.; ABREU, C.A. Os métodos de análise química do sistema IAC de análise de solo no contexto nacional. In: RAIJ, B.van; ANDRADE, J.C.; CANTARELLA, H.; QUAGGIO, J.A. (Ed.). Análise química para avaliação da fertilidade de solos tropicais. Campinas: IAC. 2001. p.5-39.

SCULL, P.; FRANKLIN, J.; CHADWICK, O.A.; McARTHUR, D. Predictive soil mapping: a review. Progress in Physical Geography, v.27, p.171-197, 2003.

SHEPHERD, K.D.; WALSH, M.G. Development of reflectance spectral libraries for characterization of soil properties. Soil Science Society of America Journal, v.66, p.988-998, 2002.

SOUSA JUNIOR, J.G. Sensoriamento remoto e sistema de informações geográficas na caracterização de solos e quantificação de seus atributos. 2005. 141p. Dissertação (Mestrado em Solos e Nutrição de Plantas) - Escola Superior de Agricultura "Luiz de Queiroz", Universidade de São Paulo, Piracicaba.

STATISTICAL ANALYSIS SYSTEM. SAS 8.02. Cary, 1999. (5 CD-ROM)

SULLIVAN, D.G.; SHAW, J.N.; RICKMAN, D.; MASK,P.L.; LUVALL, J.C. Using remote sensing data to evaluate surface soil properties in Alabama Ultisols. Soil Science, v.170, n.12, p.954-968, 2005.

UDELHOVEN, T.; EMMERLING, C.; JARMER, T. Quantitative analysis of soil chemical properties with diffuse reflectance spectroscopy and partial least-square regression: a feasibility study. Plant and Soil, v.251, n.2, p.319-329, 2003.

VISCARRA ROSSEL, R.; WALVOORT, D.J.J.; McBRATNEY, A.B.; JANIK, L.J.; SKJEMSTAD, J.O. Visible, near infrared, mid infrared or combined diffuse reflectance spectroscopy for simultaneous assessment of various soil properties. Geoderma, v.131, n.1/2, p.59-75, 2006. 